\title{
Prehospital dexamethasone administration in children with croup: a medical record review
}

This article was published in the following Dove Press journal:

Open Access Emergency Medicine

\author{
Samina $A i^{1,2}$ \\ Aaron Moodley ${ }^{3}$ \\ Amrita Bhattacharjee ${ }^{4}$ \\ Eddie Chang ${ }^{5}$ \\ Allison Kabaroff ${ }^{5}$ \\ Kevin Lobay ${ }^{5}$ \\ Dominic Allain ${ }^{6}$ \\ 'Department of Pediatrics, Faculty \\ of Medicine \& Dentistry, University \\ of Alberta, Edmonton, AB, Canada; \\ ${ }^{2}$ Women and Children's Health \\ Research Institute, Edmonton, AB, \\ Canada; ${ }^{3}$ Department of Pediatrics, \\ Faculty of Medicine, University of \\ Saskatchewan, Saskatoon, SK, Canada; \\ ${ }^{4}$ Faculty of Medicine \& Dentistry, \\ University of Alberta, Edmonton, $A B$, \\ Canada; ${ }^{5}$ Department of Emergency \\ Medicine, Faculty of Medicine \& \\ Dentistry, University of Alberta, \\ Edmonton, AB, Canada; ${ }^{6}$ Department \\ of Pediatrics, Faculty of Medicine, \\ Dalhousie University, Halifax, NS, \\ Canada
}

Objectives: Croup is one of the most common childhood respiratory illnesses. Early dexamethasone administration in croup can improve patient outcomes. The objective of this study was to assess the clinical impact of prehospital administration of dexamethasone to children with croup. Methods: A medical record review that included children between 6 months and 6 years, who were brought via emergency medical services (EMS) to the emergency department (ED) with a final diagnosis of croup, between January 2010 and December 2012, was conducted. Data were collected regarding prehospital management and ED management, length of stay (LOS), final disposition, and patient demographics.

Results: A total of 188 patients with an ED diagnosis of croup were enrolled, 35.1\% (66/188) of whom received a prehospital diagnosis of croup. The mean age of the participants was $32.96 \pm 17.18$ months and $10.6 \%(20 / 188)$ were given dexamethasone in the prehospital setting by EMS, while 30.3\% (57/188) were given epinephrine nebulizations. Out of the 66 patients with a prehospital diagnosis of croup, $10.6 \%$ (7/66) were given dexamethasone by EMS. In ED, dexamethasone was administered to $88.3 \%$ (166/188) while $29.8 \%$ of participants $(56 / 188)$ received epinephrine nebulizations. There was no significant difference in ED LOS between those who received prehospital dexamethasone $(2.6 \pm 1.6$ hours, $n=18)$ and those who did not (3.3 \pm 2.7 hours, $n=159)(P=0.514)$. The number of in-hospital epinephrine doses per patient was significantly influenced by the administration of prehospital dexamethasone $(P=0.010)$.

Conclusions: Prehospital administration of dexamethasone results in less ED epinephrine use and may reflect dexamethasone's positive influence on the severity and short-term persistence of croup symptoms.

Keywords: croup, emergency medical services, paramedic, pediatrics, steroids

\section{Introduction}

Croup (acute viral laryngotracheobronchitis) is one of the most common childhood respiratory illnesses, affecting more than 80,000 Canadian children per year. ${ }^{1}$ It carries a significant health care burden, with approximately 20,000 hospital admissions each year in the United States, at an estimated cost of $\$ 20$ million. ${ }^{2,3}$ It is also one of the most frequent causes of acute respiratory distress in young children, ${ }^{4}$ necessitating frequent emergency department (ED) visits and hospitalizations. ${ }^{1,2}$ Croup is also the most common cause of airway obstruction in children 6 months to 6 years old, ${ }^{5}$ and occurs in $3 \%$ of children under six years of age ${ }^{6}$ Croup constitutes a substantial proportion of all pediatric hospitalizations, accounting for $2 \%$ of all pediatric tertiary care hospital admissions. $^{7}$
Correspondence: Samina Ali

Department of Pediatrics, 3-583

Edmonton Clinic Health Academy,

II 405 - 87 Avenue, Edmonton,

AB T6G IC9, Canada

$\mathrm{Tel}+\mathrm{I} 7802485575$

Fax +I 8887758876

Email sali@ualberta.ca 
Early dexamethasone administration is associated with reduction in croup illness severity. ${ }^{8-10}$ Dexamethasone use in croup can reduce admission rates, length of stay (LOS), ${ }^{11}$ transfers to the intensive care unit, number of intensive care unit days, number of intubations ${ }^{12-14}$ as well as return visits to the ED. ${ }^{14,15}$ Dexamethasone also reduces the frequency of administration of nebulized epinephrine, a treatment reserved primarily for acute moderate-severe respiratory distress. ${ }^{7,16,17}$ These proven benefits all, in turn, lessen both the clinical and the financial burden of this disease. A seminal Canadian study ${ }^{18}$ has demonstrated an average saving of $\$ 21$ per case of croup in which dexamethasone was used, and a second study ${ }^{19}$ estimated a saving of $\$ 940$ per admission avoided in patients with croup. Considering these patient and systemlevel benefits, dexamethasone is now accepted as a standard treatment for the management of acute croup in children. ${ }^{1,4,10}$

To our knowledge, the impact of dexamethasone administration in the prehospital setting has not been studied. Prehospital Emergency Medical Services paramedic teams in Edmonton, Alberta (Canada) have had the mandate to administer dexamethasone $(0.6 \mathrm{mg} / \mathrm{kg}$ orally or via intramuscular injection, up to a maximum of $8 \mathrm{mg}$, at the time of study) to children with croup since $2009,{ }^{20}$ however there are no studies assessing the clinical impact of this policy. There is a need to assess the clinical impact of dexamethasone administration by prehospital care workers, in order to determine if it has an effect on clinically relevant patient outcomes for children with croup. The objectives of this study were to 1) assess the clinical impact of prehospital administration of dexamethasone to children with croup; and 2) compare clinical outcomes of these patients to those who did not receive their first dose of dexamethasone via the Emergency Medical Services (EMS) providers.

\section{Methods}

This study was conducted as a retrospective medical record review. This study followed recommended methodological guidelines for emergency medicine medical record reviews, as published by Worster and Haines and Gilbert et al. ${ }^{21,22}$ The review included children between 6 months and 6 years of age $^{5}$ who were brought via EMS to the Stollery Children's Hospital ED (Edmonton, Alberta) between January 1, 2010 and December 31, 2012, with a final International Classification of Disease (ICD)-10 diagnosis of croup per ED discharge diagnosis. Participants were excluded if they had had steroid therapy in the preceding 2 weeks, or a prior visit to an ED due to croup in the past 7 days (as we wished to capture first time presentations of the current illness and avoid double representation of the same child). To capture seasonal and temporal variations in the presentation of respiratory illnesses, all consecutive medical records of patients who presented to the ED during the period January 1, 2010 and December 31, 2012 were studied, provided they met the inclusion and exclusion criteria. For each case, data were collected on prehospital presentation and management, ED presentation and management, ED LOS and final disposition, as well as patient demographic characteristics.

The study's specific data collection tool was created with expert team input, including pediatric emergency medicine, emergency medical services, and pediatric pulmonology representation. This data abstraction tool was piloted to assess the feasibility of the planned investigation, reliability of the data abstraction instrumentation, effectiveness of protocols, availability of data and to identify any sampling concerns, as recommended by Gearing et al. ${ }^{23}$

Prehospital presentation and management data as well as level of training of EMS personnel were abstracted from paper-based EMS patient care records which are routinely appended to the hospital records for each patient brought in via EMS. ED presentation and management, ED stay parameters and demographics data was abstracted from the paper-based hospital records.

Abstractor blinding to study hypothesis was not possible as one of the co-investigators was performing part of the data abstraction. To ensure performance monitoring, the research team met periodically to resolve issues and maintain consistency in abstraction. This study was approved by the University of Alberta Health Research Ethics Board (Pro00038099). Specifically, patient/parental consent was not required by the University of Alberta Health Research Ethics Board, as medical record review studies are typically exempt from requiring explicit consent at our institution. Finally, in order to respect patient confidentiality, only de-identified data was used in our study.

Statistical analysis was performed using SPSS statistical software (version 21, IBM Corporation, Armonk, NY, USA). The data were analyzed using descriptive statistics and the chi squared test with significance set at $P<0.05$. Means were computed for continuous data (eg, age), while proportions were calculated for dichotomous data (eg, sex). $P$-values comparing two means were computed using an unpaired $t$-test, while those between proportions were computed using Fisher's exact test. For ordinal data, the Kruskal-Wallis test was used to compare significance between two groups, while for categorical data (eg, final diagnosis) the Fisher-FreemanHalton test was employed. 


\section{Results}

\section{Medications used}

A summary of study flow can be found in Figure 1. In total, 356 medical records were reviewed. Of these, 168 medical records were excluded based on our exclusion criteria. This left 188 patient medical records that were included in this study. The mean age of the participants was 33.0 17.2 months. Details of the demographic characteristics can be found in Table 1. Overall, $10.6 \%$ of patients $(n=20)$ were given dexamethasone in the prehospital setting (ie, by EMS personnel), and $30.3 \%$ of patients $(n=57)$ were given epinephrine nebulizers by EMS. Of those patients who received prehospital epinephrine $(\mathrm{n}=57), 87.7 \%(\mathrm{n}=50)$ were given one dose and $12.3 \%(\mathrm{n}=7)$ were given two doses. Of the 188 included children, $35.1 \%(n=66)$ received a prehospital diagnosis of croup. Further details on prehospital diagnosis and management can be found in Table 2. In the ED, dexamethasone was administered to $88.3 \%$ of patients $(n=166)$, while $29.8 \%(n=56)$ received epinephrine nebulizers (Table 3). Vital signs in the prehospital setting included initial oxygen saturation (on room air) at $97.0 \%( \pm 3.9)$, heart rate of $138.6( \pm 23.9)$ beats per minute, and temperature of 37.3 $( \pm 1.1)$ degrees Celsius. ED vital signs were initial oxygen saturation (on room air) at $97.9 \%( \pm 2.3)$, heart rate of 136.3 $( \pm 22.44)$ beats per minute, and temperature of $37.1( \pm 1.3)$ degrees Celsius.

\section{Timing of interventions}

Of the patients that had a prehospital diagnosis of croup $(n=66)$, only $10.6 \%(n=7)$ received dexamethasone in the prehospital setting. The mean time from EMS arrival at home to EMS arrival at the ED was $40 \pm 22$ minutes $(n=155)$. The mean time from arrival via EMS at the ED to triage was $8 \pm 5$ minutes $(n=153)$. The mean time between ED triage to ED administration of dexamethasone was $2.0 \pm 3.7$ hours $(n=159)$, and the time between ED triage and ED administration of epinephrine nebulization was $1.9 \pm 3.3$ hours $(n=56)$. The median time from EMS arrival at the ED to ED discharge was 2.7 hours (IQR 2.0) \pm 2.1 hours ( $\mathrm{n}=149$ ). The median time from EMS arrival at home to ED discharge was 3.3 hours (IQR 2.1) (n=157).

\section{ED length of stay and disposition}

Eight of the participants were admitted to the hospital during their initial visit; further details on all patients' final dispositions can be found in Table 3. There was no significant difference in ED LOS between those who received prehospital dexamethasone (2.6 \pm 1.6 hours, $n=18)$ and those who did not $(3.3 \pm 2.7$ hours, $\mathrm{n}=159)(P=0.514)$. There was also no significant difference in ED LOS between those who received in-hospital dexamethasone $(3.3 \pm 2.7$ hours, $n=157)$ and those who did not (2.6 \pm 1.6 hours, $\mathrm{n}=20)(P=0.486)$. The rate of admission was not significantly influenced by either prehospital dexamethasone administration $(P=0.363)$, or by in-hospital dexamethasone administration $(P=0.434)$. The occurrence of an ED return visit within 7 days was not significantly influenced by prehospital $(P=0.752)$ or in-hospital ( $P=0.840)$ dexamethasone administration. Of note, the number of in-hospital epinephrine nebulization doses per patient was significantly influenced by prehospital dexamethasone administration $(P=0.010)$, with a lower number of nebulizer doses administered for patients who received prehospital dexamethasone. Of the 168 patients who did not receive dexamethasone prior to arrival in hospital, $32.7 \%$ required nebulized epinephrine in hospital. However, only $5 \%$ of the 20 patients who received prehospital dexamethasone required epinephrine nebulizers in the ED.

For patients who had a prehospital diagnosis of croup, there was no statistically significant difference in ED LOS between those who received prehospital dexamethasone and those who $\operatorname{did} \operatorname{not}(P=0.458)$. There was also no statistically significant difference in ED LOS for patients with a prehospital diagnosis of croup who received in-hospital dexamethasone, and those who did not $(P=0.458)$. In the same group of patients, the rate of admission was not influenced by inhospital $(P=0.830)$ or prehospital $(P=0.830)$ dexamethasone administration. Further, the occurrence of an ED return visit within 7 days was not significantly influenced by prehospital $(P=0.589)$ or in-hospital $(P=0.589)$ dexamethasone administration in patients with a prehospital diagnosis of croup.

\section{Discussion}

In this study, ED LOS was not influenced by prehospital dexamethasone administration. However, inability to achieve significance may be due to the small number of patients who received the medication of interest; only $10.6 \%$ of patients received dexamethasone prior to arrival at the hospital. As expected, ED LOS was not influenced by in-hospital dexamethasone administration. This is in keeping with current understanding of dexamethasone kinetics, its mechanism, and onset of action in the treatment of croup. Corticosteroid therapy works by reducing inflammation and edema in the upper airways, thereby decreasing airway resistance. ${ }^{10}$ However, the onset of action of dexamethasone is not immediate, and can take 4-6 hours to show improvement in symptoms. ${ }^{10}$ As such, we did not expect to see any immediate improvement 


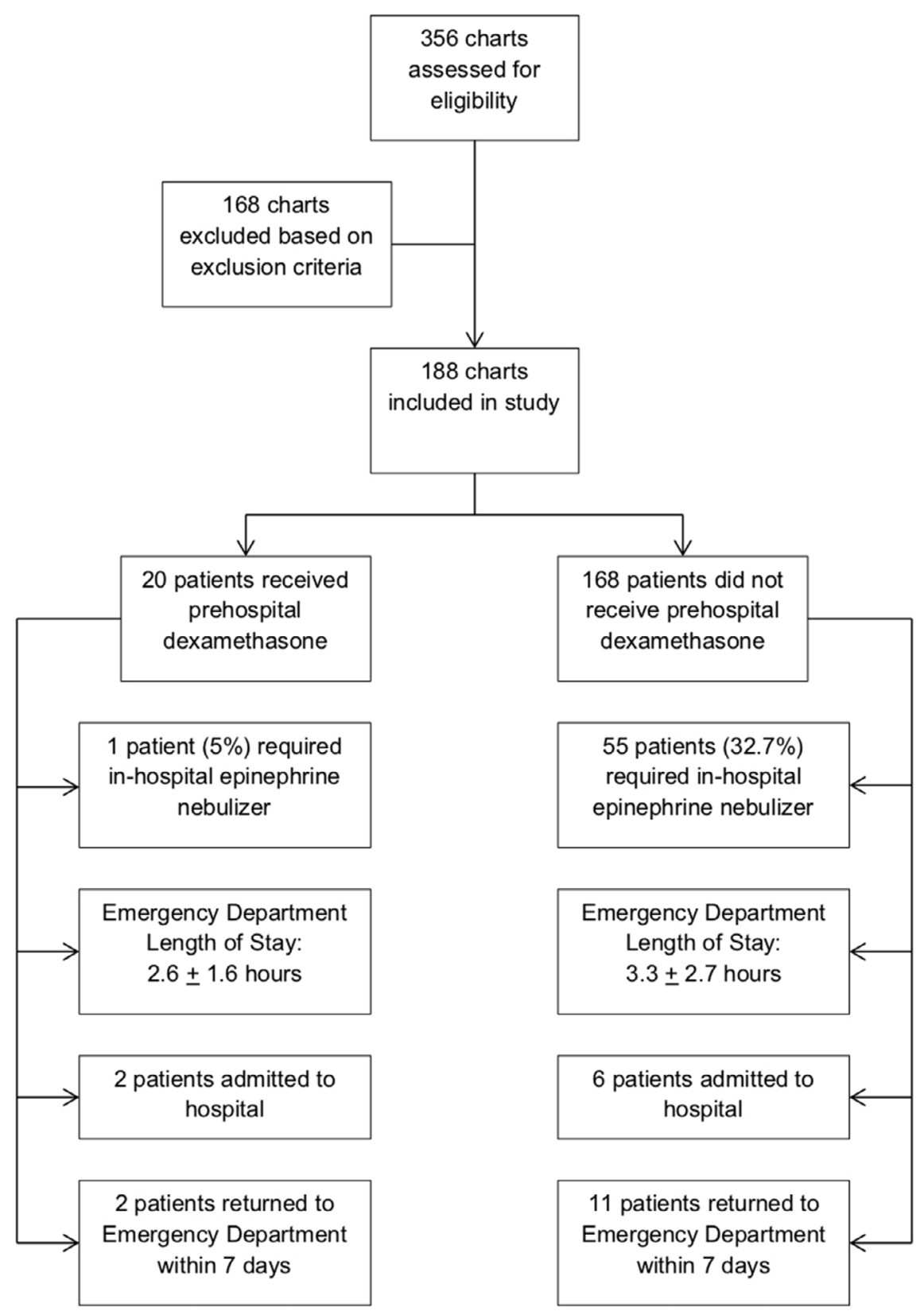

Figure I Summary of study flow.

in LOS for patients who were given dexamethasone for the first time in the ED. Interestingly, a recent single center, Australian study showed that an oral dose of $0.15 \mathrm{mg} / \mathrm{kg}$ dexamethasone offered benefit to children with croup as early as 30 minutes after administration, with lower croup score for children treated with dexamethasone, suggesting that there might be some immediate LOS benefits to administering dexamethasone early (ie, in the prehospital setting). ${ }^{24}$ This suggestion is especially important in the treatment of more severe croup, where invasive interventions (eg, admission, intubation, etc.) may be considered. However, this theory would need to be confirmed with further study.
In total, approximately one third of all patients with a final ED diagnosis of croup were given a prehospital diagnosis of croup by the EMS personnel. Of these 66 patients, only $10.6 \%$ were given dexamethasone in the prehospital setting. This contradicts local EMS protocol, which states that all patients with a diagnosis of croup are to be given dexamethasone prior to arrival at the hospital. ${ }^{25}$ On the other hand, almost one third of patients were given epinephrine nebulizations by EMS personnel prior to arrival in the hospital. It is likely that these patients who received nebulized epinephrine prior to ED arrival would have benefitted by receiving dexamethasone early, as well. While EMS personnel correctly recognized the 
Table I Demographic Characteristics of Patients

\begin{tabular}{|l|l|}
\hline Variable & $\mathbf{n}(\%)$ \\
\hline Sex $(\mathbf{n}=\mathbf{I 8 8})$ & \\
\hline Male & $13 \mathrm{I}(69.7)$ \\
\hline Female & $57(30.3)$ \\
\hline Pre-existing chronic respiratory disease (n= I87) & $43(23.0)$ \\
\hline History of prior oral steroid use (n= I86) & $26(\mathbf{I 4 . 0 )}$ \\
\hline Prior visit to local pediatric ED (n= I 88) & $81(43.1)$ \\
\hline $\begin{array}{l}\text { Any medications given by parents prior to EMS } \\
\text { arrival (n= I53) }\end{array}$ & $59(31.6)$ \\
\hline Medications administered (n=59)* & \\
\hline Acetaminophen & 22 \\
\hline Ibuprofen & 10 \\
\hline Salbutamol & 22 \\
\hline Anti-histamine & 2 \\
\hline Dextromethorphan & 2 \\
\hline Inhaled corticosteroids & 4 \\
\hline Lactulose & 1 \\
\hline Antibiotics & 2 \\
\hline Unknown/Unsure & 2 \\
\hline
\end{tabular}

Notes: *Multiple responses permitted.

Abbreviations: ED, emergency department; EMS, emergency medical services.

Table 2 Pre-hospital Diagnosis and Management $(n=188)$

\begin{tabular}{|l|l|}
\hline Variable & $\mathbf{n}(\%)$ \\
\hline Unknown & $77(4 I .0)$ \\
\hline Croup & $66(35.1)$ \\
\hline Gastroenteritis & $8(4.3)$ \\
\hline Asthma & $4(2.1)$ \\
\hline Viral Illness & $4(2.1)$ \\
\hline Bronchiolitis & $3(1.6)$ \\
\hline Other Respiratory Diagnoses* & $5(2.7)$ \\
\hline Other Non-Respiratory Diagnoses & $21(11.4)$ \\
\hline Dexamethasone administered & $20(10.6)$ \\
\hline Epinephrine nebulization administered & $57(30.3)$ \\
\hline $\begin{array}{l}\text { Number of Doses of Epinephrine Nebulizations } \\
\text { (n=57) }\end{array}$ \\
\hline \multicolumn{1}{|l}{ I } & \\
\hline 2 & $70(87.7)$ \\
\hline
\end{tabular}

Notes: *Others included diagnoses of otitis media (2), pharyngitis (2), pneumonia (1).

symptoms and diagnosed a subset of children with croup, they failed to adhere to their protocol for dexamethasone administration. This represents a missed opportunity to positively impact the course of a child's croup illness trajectory, as early dexamethasone administration is known to decrease the need for nebulized epinephrine, as well as ED LOS in other studies, and to improve outcomes overall..$^{8-15}$

The rate of admission to hospital and rate of return to ED within 7 days was not influenced by prehospital or in-hospital administration of dexamethasone. In our study, less than 5\% of patients were admitted to hospital at the index visit. It is known that admission rates for croup vary widely from $1.5 \%$
Table 3 ED management of Patients $(n=\mid 88)$

\begin{tabular}{|l|l|}
\hline Variable & $\mathbf{n}(\%)$ \\
\hline CTAS Score & \\
\hline I & $2(I . I)$ \\
\hline 2 & $67(35.6)$ \\
\hline 3 & $109(58.0)$ \\
\hline 4 & $8(4.3)$ \\
\hline 5 & $\mathrm{I}(0.5)$ \\
\hline Dexamethasone administered & $166(88.3)$ \\
\hline Route of dexamethasone administration & \\
\hline PO & $165(87.8)$ \\
\hline \multicolumn{1}{|c|}{ Not reported/unclear documentation } & $23(12.2)$ \\
\hline Epinephrine nebulization administered & $56(29.8)$ \\
\hline $\begin{array}{l}\text { Number of epinephrine doses administered } \\
\text { (n=56) }\end{array}$ & \\
\hline I & $35(62.5)$ \\
\hline 2 & $14(25.0)$ \\
\hline 3 & $7(12.5)$ \\
\hline ED Discharge Disposition (n= I88) & \\
\hline Discharged Home & $180(95.7)$ \\
\hline Admitted & $8(4.3)$ \\
\hline Transferred to ICU & $0(0.0)$ \\
\hline Died & $0(0.0)$ \\
\hline Return visit to pediatric ED within 7 days & $13(6.9)$ \\
\hline Return Visit Disposition (n= I3) & \\
\hline Admitted & $2(15.4)$ \\
\hline Readmitted & $\mathrm{I}(7.7)$ \\
\hline Left without being seen & $2(15.4)$ \\
\hline Discharged home & $8(61.5)$ \\
\hline
\end{tabular}

Abbreviations: CTAS, Canadian Triage and Acuity Scale; ED, emergency department; ICU, intensive care unit; PO, per os (by mouth).

to $31 \%$, depending on the severity of the disease and hospital admission practices. ${ }^{10,15}$ Like other studies that looked at rates of hospital admissions in children with croup, we expected the rate of admission in our study to be lower in the group that received prehospital dexamethasone compared to those who did not. The inability to reach significance may, at least in part, be attributed to the large difference in size between the two groups.

Our study found that the number of in-hospital epinephrine nebulization doses per patient was significantly influenced by the administration of prehospital dexamethasone. Almost one third of patients who were not given dexamethasone by EMS required nebulized epinephrine in-hospital. In contrast, only one patient (out of 20) who was given dexamethasone by EMS required in-hospital epinephrine nebulization. This supports our understanding of the onset of action of dexamethasone as somewhat delayed, allowing for patients who were given dexamethasone by EMS to be less likely to require an epinephrine nebulization while in the ED. Given that overcrowding of EDs is an ongoing issue in recent years, ${ }^{26}$ any intervention that positively impacts ED flow is a welcome addition to ED practice. Each dose of 
nebulized epinephrine requires 15 minutes for administration; this means that a health care professional, likely a nurse or a respiratory therapist must be present for part or all of the 15 minutes while the patient receives the proper dose of epinephrine. Sometimes, patients require multiple doses of nebulized epinephrine, and each additional dose is another period of time that the health care professional is unavailable to tend to other patients or matters. Out of the 55 patients in our study who were not given prehospital dexamethasone and required nebulized epinephrine in the ED, over one third required more than one dose. As well, our study showed that if patients with croup did not receive dexamethasone from EMS prior to arrival at the hospital, they had to wait an average of another 2 hours before they were given their first dose of dexamethasone. Once again, based on the somewhat delayed onset of action of dexamethasone, this means that patients may have been more likely to receive nebulized epinephrine while waiting for dexamethasone to take effect, which in turn resulted in them occupying a bed for a longer period and a longer ED LOS overall.

A limitation of this study was the small number of included patients, overall, and in particular, those who received the intervention of interest: prehospital dexamethasone. Lack of recording of croup severity scores also limited our capacity to comment on the relationship of LOS and dexamethasone administration with severity of illness. Further, as this was a retrospective medical record review, it was subject to all of the inherent limitations of this methodology.

Prehospital administration of dexamethasone results in less ED epinephrine use and may reflect dexamethasone's positive influence on the severity and short-term persistence of croup symptoms. A future larger study, with a greater sample size, may help to confirm some of the findings that did not attain statistical significance in this smaller study (eg, ED LOS). Contrary to current guidelines, few patients with a prehospital diagnosis of croup received dexamethasone by EMS personnel. This represents a missed opportunity to administer dexamethasone early and possibly decrease the severity of the patients' disease.

\section{Acknowledgments}

We would like to acknowledge Ms Nadia Dow for research coordination assistance, Ms Melissa Gutland for administrative support for this study, the Clinical Research Informatics Core (Women and Children's Health Research Institute) for database support, and Dr Maryna Yaskina, PhD (Women and Children's Health Research Institute) for statistical support.
Dr Aaron Moodley secured a Women and Children's Health Research Institute Trainee Research Grant to support this work (2013-2015).

The abstract of this paper was presented at the Canadian Association of Emergency Physicians Annual Meeting (Quebec City, Quebec, Canada) in June 2016 as a poster presentation with interim findings. The poster's abstract was published in "Poster Abstracts" in the Canadian Journal of Emergency Medicine. ${ }^{27}$

\section{Disclosure}

The authors report no conflicts of interest in this work.

\section{References}

1. Bjornson CL, Johnson DW. Croup in children. CMAJ. 2013;185(15): 1317-1323.

2. Henrickson KJ, Kuhn SM, Savatski LL. Epidemiology and cost of infection with human parainfluenza virus types 1 and 2 in young children. Clin Infect Dis. 1994;18(5):770-779.

3. Prendergast M, Jones JS, Hartman D, Jeffrey SJ, David H. Racemic epinephrine in the treatment of laryngotracheitis: can we identify children for outpatient therapy? Am J Emerg Med. 1994;12(6):613-616.

4. Bjornson CL, Johnson DW. Croup. Lancet. 2008;371(9609):329-339.

5. Mandal A, Kabra SK, Lodha R. Upper airway obstruction in children. Indian J Pediatr. 2015;82(8):737-744.

6. Denny FW, Murphy TF, Clyde WA, Collier AM, Henderson FW. Croup: an 11-year study in a pediatric practice. Pediatrics. 1983;71(6): 871-876.

7. Johnson DW, Jacobson S, Edney PC, Hadfield P, Mundy ME, Schuh $\mathrm{S}$. A comparison of nebulized budesonide, intramuscular dexamethasone, and placebo for moderately severe croup. $N$ Engl J Med. 1998;339(8):498-503.

8. Jaffe DM. The treatment of croup with glucocorticoids. $N$ Engl J Med. 1998;339(8):553-555.

9. Cruz MN, Stewart G, Rosenberg N, Gail S, Norman R. Use of dexamethasone in the outpatient management of acute laryngotracheitis. Pediatrics. 1995;96(2 Pt 1):220-223.

10. Russell KF, Liang Y, O'Gorman K, Johnson DW, Klassen TP. Glucocorticoids for croup (Review). Cochrane Database Syst Rev. 2011;1.

11. Kunkel NC, Baker MD. Use of racemic epinephrine, dexamethasone, and mist in the outpatient management of croup. Pediatr Emerg Care. 1996;12(3):156-159.

12. Kairys SW, Olmstead EM, O'Connor GT. Steroid treatment of laryngotracheitis: a meta-analysis of the evidence from randomized trials. Pediatrics. 1989;83(5):683-693.

13. Tibballs J, Shann FA, Landau LI. Placebo-controlled trial of prednisolone in children intubated for croup. Lancet. 1992;340(8822):745-748.

14. Geelhoed GC, Turner J, MacDonald WB. Efficacy of a small single dose of oral dexamethasone for outpatient croup: a double blind placebo controlled clinical trial. BMJ. 1996;313(7050):140-142.

15. Rizos JD, Digravio BE, Sehl MJ, Tallon JM. The disposition of children with croup treated with racemic epinephrine and dexamethasone in the emergency department. J Emerg Med. 1998;16(4):535-539.

16. Ausejo M, Saenz A, Pham B, et al. The effectiveness of glucocorticoids in treating croup: meta-analysis. BMJ. 1999;319(7210):595-600.

17. Klassen TP, Feldman ME, Watters LK, Sutcliffe T, Rowe PC. Nebulized budesonide for children with mild-to-moderate croup. $N$ Engl J Med. 1994;331(5):285-289.

18. Bjornson CL, Klassen TP, Williamson J, et al. Pediatric Emergency Research Canada Network. A randomized controlled trial of a single dose of dexamethasone in croup. N Engl J Med. 2004;351(13):1306-1313. 
19. Ledwith CA, Shea LM, Mauro RD, Lytitia MS, Robert DM. Safety and efficacy of nebulized racemic epinephrine in conjunction with oral dexamethasone and mist in the outpatient treatment of croup. Ann Emerg Med. 1995;25(3):331-337.

20. Health Quality Council of Alberta. Review of Operations of Ground Emergency Medical Services in Alberta. Available from: http://www. health.alberta.ca/documents/EMS-Review-HQCA-2013.pdf. Accessed June 28, 2018.

21. Worster A, Haines T. Advanced statistics: understanding medical record review (MRR) studies. Acad Emerg Med. 2004;11(2):187-192.

22. Gilbert EH, Lowenstein SR, Koziol-McLain J, Barta DC, Steiner J. Chart reviews in emergency medicine research: where are the methods? Ann Emerg Med. 1996;27(3):305-308.

23. Gearing RE, Mian IA, Barber J, Ickowicz A. A methodology for conducting retrospective chart review research in child and adolescent psychiatry. J Can Acad Child Adolesc Psychiatry. 2006;15(3):126-134.
24. Dobrovoljac M, Geelhoed GC. How fast does oral dexamethasone work in mild to moderately severe croup? A randomized double-blinded clinical trial. Emerg Med Australas. 2012;24(1):79-85.

25. Alberta Health Services. EMS Medical Control Protocol for the management of Pediatric Stridor. Available from: https://www.ahsems.com/ public/protocols/templates/desktop/\#set/12/browse/4590/view/38057/ Algorithm . Accessed June 25, 2018.

26. Global News. Canadian Healthcare Struggles to Find a Cure for Hallway Medicine. Available from: http://globalnews.ca/news/3406842/ canadian-health-care-struggles-to-find-a-cure-for-hallway-medicine/. Accessed June 25, 2018.

27. Ali S, Moodley A, Bhattacharjee A, et al. P005: Pre-hospital dexamethasone administration in children with croup: the Edmonton experience. CJEM. 2016;18(Suppl S1):S80.
Open Access Emergency Medicine

\section{Publish your work in this journal}

The Open Access Emergency Medicine is an international, peerreviewed, open access journal publishing original research, reports, editorials, reviews and commentaries on all aspects of emergency medicine. The manuscript management system is completely online and includes a very quick and fair peer-review system, which is all

\section{Dovepress}

easy to use. Visit http://www.dovepress.com/testimonials.php to read real quotes from published authors. 